\author{
Revista Calidad en la Educación Superior \\ Programa de Autoevaluación Académica \\ Universidad Estatal a Distancia, Costa Rica \\ ISSN 1659-4703 \\ revistacalidad@uned.ac.cr
}

\title{
UN ACERCAMIENTO AL APRENDIZAJE DE LAS PERSONAS ADULTAS: APUNTES PARA RE-PENSAR LA MEDIACIÓN PEDAGÓGICA
}

\author{
A LEARNING APPROACH FOR ADULT PEOPLE: NOTES TO RETHINK THE \\ PEDAGOGICAL MEDIATION
}

Wendy Obando Leiva ${ }^{1}$ wobando@uned.ac.cr
Marianela Salas Soto msalas@uned.ac.cr $^{2}$ Universidad Estatal a Distancia, Costa Rica

\author{
Volumen 6, número 1 \\ Mayo 2015 \\ Pp. $119-149$
}

Recibido: 06 de marzo, 2015

Aprobado: 27 de marzo, 2015

\footnotetext{
${ }^{1}$ Wendy Obando Leiva, Máster en Psicología Educativa, Licenciada en Psicología y Bachiller en Enseñanza. Es profesora capacitadora en el Centro de Capacitación a Distancia de la UNED y docente e investigadora en la Universidad de Costa Rica.

2Marianela Salas Soto, Máster en Planificación Curricular y Licenciada en Educación. Es coordinadora del Centro de Capacitación a Distancia de la UNED y docente en la Universidad de Costa Rica.
} 


\section{Resumen}

La Universidad Estatal a Distancia (UNED), como institución dedicada a ofrecer una educación que promueva el desarrollo integral de personas que, en su mayoría, se encuentran en la etapa de adultez se fundamenta en un modelo pedagógico que considera dentro de sus principios características propias del nivel cognitivo, afectivo y social de las personas aprendientes que están vinculadas a los procesos de enseñanza-aprendizaje. En sus inicios, la población de esta universidad estaba representada por personas ubicadas dentro de la mediana edad, pero al pasar los años el porcentaje de estudiantes universitarios adultos jóvenes (menores de treinta años) ha ido aumentando. Ante esta situación interesa recoger evidencias de la existencia de diferencias específicas en la educación durante esta etapa, con el fin de reconocer los retos que representa la mediación de experiencias para la educación superior con dicha población.

Palabras claves: educación superior; educación a distancia; mediación pedagógica; ciclo de vida; etapa adulta

\section{Abstract}

The Universidad Estatal a Distancia (UNED) is an institution committed to the education of adults based on a pedagogical model which principles embrace aspects that considers within its principles, own cognitive, affective and social level of the learners who are involved in the teaching-learning.

At the foundation of this university, its population was represented by middle aged students; however, along the years, the percentage of young adults (under 30 years) that are part of its student body, has increased. Having noticed this peculiarity, it is important to gather evidence regarding existing specific differences in the education at this stage, and in that way, identify the challenges that the mediation of experiences for such population represents for the tertiary education.

Keywords: tertiary education; distance education; pedagogical mediation; lifecycle; adulthood 
Las diferentes etapas del desarrollo (infancia, adolescencia, juventud, adultez y madurez) tienen características cognitivas y sociopersonales propias, las cuales inciden en la potenciación del aprendizaje en la medida en la que el contexto biopsicosocial de la persona es considerado como parte del proceso de mediación.

Un abordaje profundo sobre el papel que ocupa la etapa de desarrollo en el aprendizaje de los seres humanos requeriría forzosamente una lectura interdisciplinaria, ya que incluiría un intenso análisis desde puntos de vista epistemológicos, antropológicos, sociológicos, psicológicos, pedagógicos, entre otros. En general, las investigaciones alrededor de este tema todavía presentan un debate sobre la diferenciación del aprendizaje en las etapas del ciclo vital; pero, si se consideran los aportes pedagógicos al respecto, se podrá comprobar que cuando se habla del carácter específico de la educación de personas adultas, de una $u$ otra forma, el problema que se plantea presenta dos concepciones globales que rivalizan entre sí (Medina, 2000):

1) Como una aplicación adaptativa de la pedagogía general a las personas adultas, en torno a pautas generales que son aplicables a todas las edades.

2) Como un saber educativo específico e idiosincrático en el campo de la pedagogía, que requeriría un conjunto de prácticas educativas diferenciadas.

La posición presentada en este artículo parte de que las diferencias que pudieran existir entre el aprendizaje de las personas adultas y el de los niños y adolescentes dependerá en gran medida del significado que, desde el punto de vista psicológico y social, tenga el hecho de ser una persona adulta y del modo en que se concrete el carácter optimizante de esta acción educativa.

El presente texto pretende brindar aportes desde una perspectiva psicopedagógica, al realizar un recorrido por algunos elementos fundamentales 
concernientes a las características biopsicosociales de las personas adultas, con la intención de promover la reflexión sobre las implicaciones y los retos para la mediación pedagógica durante esta etapa; lo anterior sin llegar a abarcar todo el análisis interdisciplinario que se podría realizar.

\section{La población aprendiente: las personas adultas}

La UNED nació con la intención de ofrecerle a la sociedad costarricense una formación amplia, holística e interdisciplinaria, orientada a brindar espacios de profesionalización ante el aumento de la demanda de educación superior en distintas regiones del país. Hoy cuenta con treinta y cuatro centros universitarios y tres subsedes a lo largo y ancho del territorio nacional, en los que se encuentra matriculado un total de 30238 estudiantes, de los cuales 19281 son mujeres y 10 957 son hombres ${ }^{3}$ (ver gráfico 1):

\section{Gráfico 1. Número de estudiantes por sexo matriculados en la UNED}

(años 2012-2014)

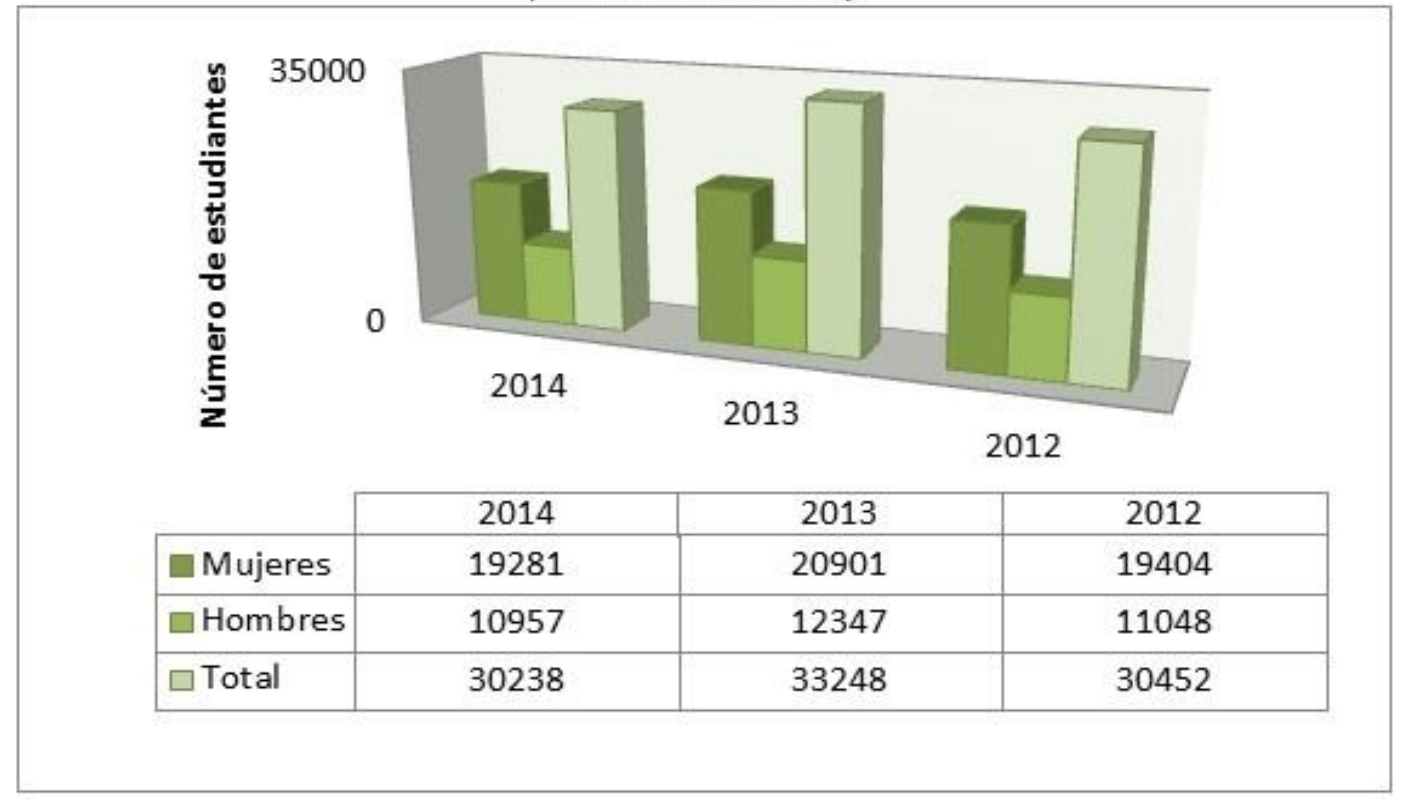

Fuente: SAE (Centro de Investigación y Evaluación Institucional [CIEI], 2014)

\footnotetext{
${ }^{3}$ El informe del Sistema de Administración de Estudiantes (SAE) presenta a una persona sin ubicar en la categoría sexo.
} 
De la totalidad de personas matriculadas en el año 2014 , el $25,2 \%$ se encuentra inscrito en el Centro Universitario de San José, 7,8\% en el de Heredia, $7,7 \%$ en el de Cartago, $6,4 \%$ en Alajuela y el restante $52,9 \%$ se distribuye heterogéneamente entre los demás centros del país.

Un alto porcentaje de esta población es adulta joven, ${ }^{4}$ así $62,8 \%$ de los y las estudiantes de grado tiene menos de treinta años de edad, $26,2 \%$ se encuentra entre los treinta y los cuarenta años y tan solo un $11 \%$ de las personas aprendientes tiene una edad superior a cuarenta. Esta tendencia se repite en la población matriculada durante los años 2012 y 2013 (ver gráfico 2):

\section{Gráfico 2. Número de estudiantes por rango de edad matriculados en grado (UNED)}

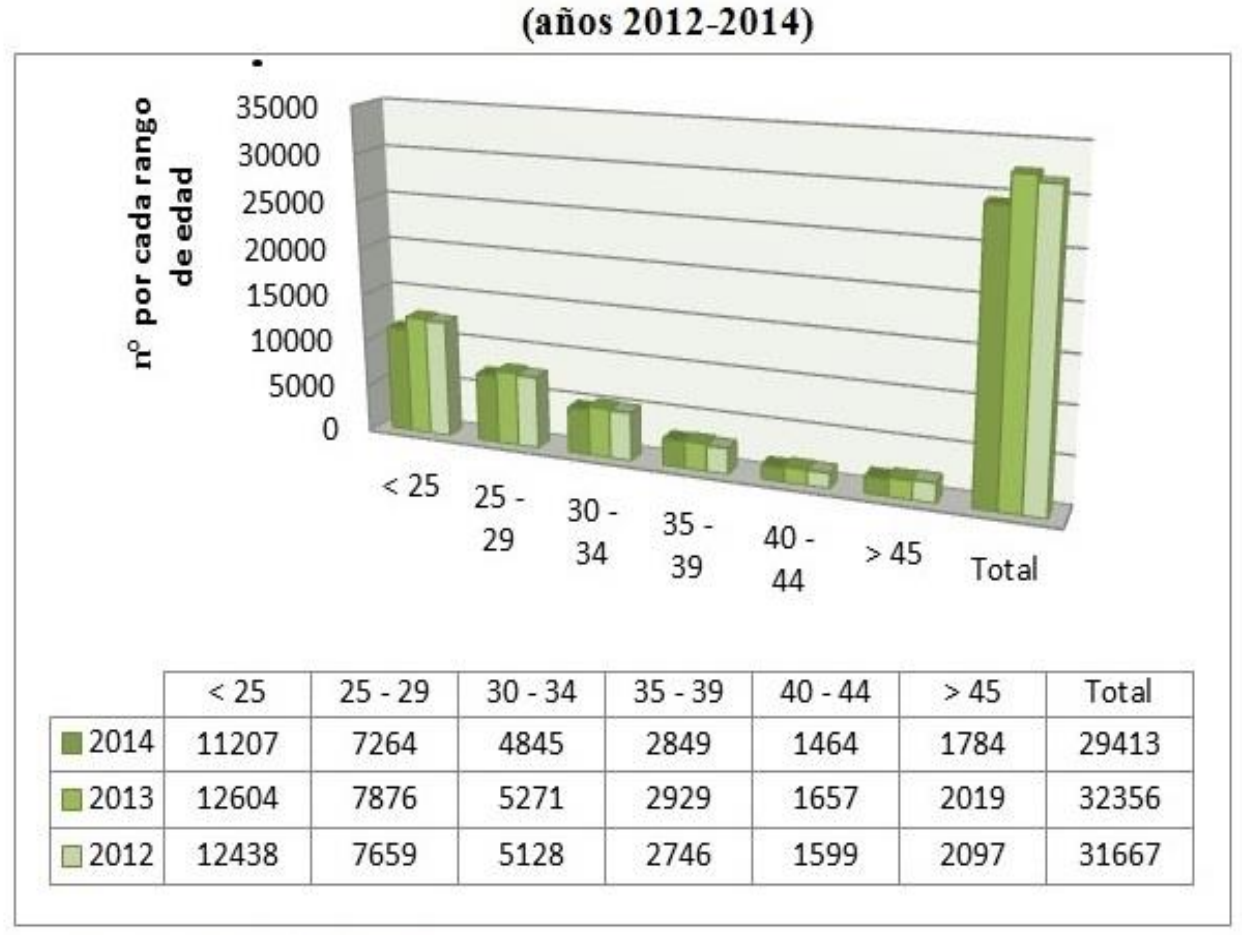

Fuente: SAE (CIEI, 2014)

En los estudios de posgrado se da un comportamiento diferente, ya que, de 776 estudiantes matriculados, el $21,6 \%$ es menor de treinta años, $50,3 \%$ se encuentra

\footnotetext{
${ }^{4}$ El informe del SAE reporta cincuenta personas sin ubicar en la categoría edad.
} 
entre los treinta y cuarenta años y $28,1 \%$ es mayor de cuarenta años (ver gráfico 3):

Gráfico 3. Número de estudiantes por rango de edad matriculados en posgrado (UNED) (años 2012-2014)

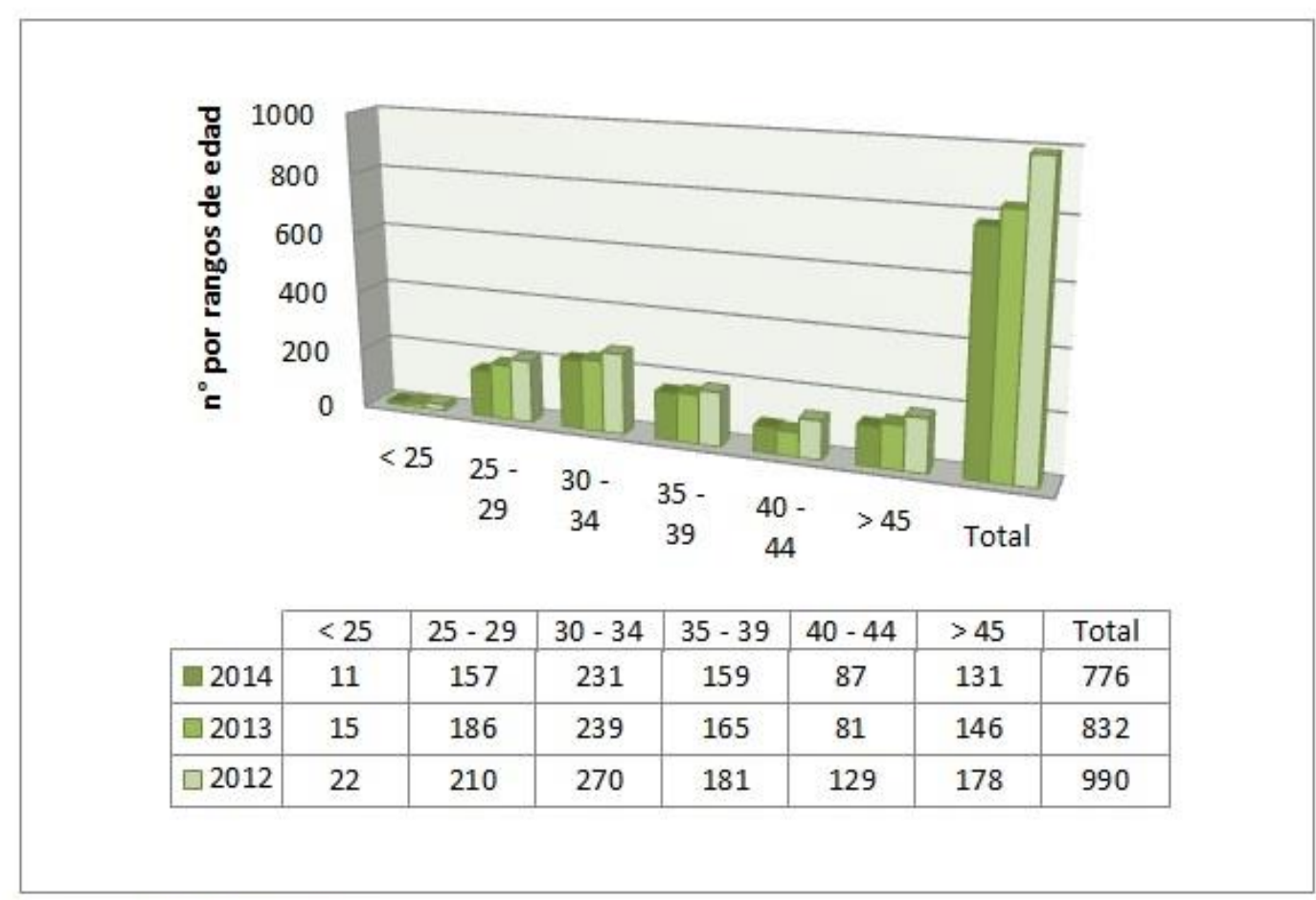

Fuente: SAE (CIEI, 2014)

A partir de esta información se aprecia que, en resumen, un gran porcentaje de estudiantes de la UNED son adultos y adultas jóvenes (62,8\% entre dieciocho y treinta años de edad) -en mayor porcentaje mujeres $(63,8 \%$ mujeres versus $36,2 \%$ hombres) - matriculados en mayor porcentaje en el Centro Universitario de San José $(25,2 \%)$ y en menor número en los diferentes centros universitarios del país $(74,8 \%)$.

Precisamente por estas particularidades se considera relevante realizar un recorrido por el aporte de diversos autores y autoras que se han dado a la tarea de enfatizar los rasgos más fundamentales de esta etapa, al dar a conocer el 
contexto en el que se desarrolla la vida adulta, las demandas de dicho contexto en su educación, las características propias del aprendizaje en personas adultas y otras cuestiones importantes que deben tenerse en cuenta para promover y mejorar este proceso.

\section{El contexto de la persona adulta y la educación}

La sociedad actual es cambiante y desafiante, traslada constantemente al ser humano por senderos desconocidos de incertidumbre $y$, a la vez, influye profundamente en diferentes espacios de su subjetividad: trabajo, familia, ocio, espiritualidad, etc., lo cual le incita a adoptar por determinados estilos de vida (Capra, 1992; McLaren, 1997; Morin, 2006; Najmanovich, 2005).

Las personas adultas deben ajustarse a este permanente cambio y tomar decisiones cotidianas para sobrevivir manteniendo determinada calidad de vida; no obstante, la mayor parte de los sistemas educativos mantiene una homodinamia (dinámica sin cambios) sin poder dar una réplica adecuada a la sociedad cambiante en la que se desenvuelven. Es por ello que la educación actual no debería perseguir la enseñanza para un mundo conocido, sino que, por el contrario, debería generar espacios de aprendizaje para promover la convivencia en un mundo caracterizado por el cambio permanente y que da lugar a situaciones imprevisibles.

Villanueva (2001) ha señalado algunos de los factores característicos de esta sociedad en la que la persona se desarrolla dialécticamente:

1. Productividad en aumento, como consecuencia de la automatización, informatización y reducción de empresas marginales

2. Reducción del tiempo de trabajo y aumento del ocio

3. Mayor frecuencia de las actividades económicas terciarias (servicios) y las cuaternarias (investigación, formación, cultura). 
4. Sociedad de masas con la aparición de las megalópolis, con abundancia de hiperorganización y burocratización, al mismo tiempo que la falta de equipamiento

5. Desarrollo del anonimato social, de la inadaptación y del conformismo social, y la aparición de una contrasociedad algunas veces integrada, otras veces ofensiva

6. Desarrollo de los conceptos del disfrute, de lo laico, de lo humanista y del confort personal como conceptos esenciales

El espíritu de esta sociedad postindustrial hace que «la producción del conocimiento» —su creación, su difusión y su apropiación- ocupe un lugar primordial en la cultura y la educación, pues junto a este conocimiento aparece la innovación. A la vez, florece el fenómeno de la obsolescencia cultural (los saberes y sus aplicaciones mueren antes de su aparición), lo que obliga a luchar contra el envejecimiento prematuro del saber y de la cultura.

Dadas estas características socioculturales, resulta fundamental un protagonismo educativo dirigido a ayudar a la persona a pensar, a enseñar a aprender, a inculcar amor profundo por la idea de conocer; más que a dar información, saber dónde buscarla y cómo seleccionarla e interpretarla; debe ayudar a generar nuevos conocimientos (Escotet, 1990, citado por Villanueva, 2001).

Dichos cambios técnico-culturales también manifiestan la necesidad de reconstruir otro tipo de educación en el que la innovación permita el refrescamiento constante de los saberes: "La clave de este objetivo se halla en la participación, que permite al estudiante examinar los lugares recónditos del conocimiento, poner en tela de juicio su importancia y significado y replantear, combinar y organizar sus ideas, siempre que ello sea necesario» (Villanueva, 2001, p.166).

Las vicisitudes de la época también convocan a re-pensar la educación, de manera que trascienda sus objetivos netamente pedagógicos hacia otras tareas 
fundamentales; lo anterior debido a que resulta un componente absolutamente indispensable para construir, en cada sociedad nacional, un desarrollo humano digno. En palabras de Picón (2013):

El desarrollo humano implica desarrollo económico y desarrollo social articulados, que sirvan a las personas, a las comunidades y las sociedades nacionales para el mejoramiento de sus condiciones y calidad de vida. Implica también el ejercicio pleno de una ciudadanía intercultural, histórica, económica, política y organizacional, con miras a lograr niveles crecientes de bienestar y de felicidad (p.148).

Para alcanzar esta meta el principal desafío que se presenta es que la educación superior oriente actitudinal y vocacionalmente el tránsito hacia el mundo del trabajo, pero también hacia una convivencia armoniosa en los distintos ámbitos de la vida cotidiana: familias, amigos y amigas, comunidad, espiritualidad, etc. Es decir, lo académico no se encuentra desvinculado de la cotidianidad ni de las tareas propias que como ciudadano y ciudadana se deben desarrollar.

\section{Características del aprendizaje en la etapa adulta: mejorando las experiencias}

Al considerarse diversas investigaciones psicológicas sobre los cambios ocasionados durante el desarrollo del ciclo vital y partiendo de conocimientos pedagógicos concretos, se puede afirmar que existen evidencias que fundamentan algunos aspectos del carácter específico de la educación de personas adultas como un tipo de educación propia que se diferencia de lo que habitualmente se realiza en las escuelas con la niñez y la adolescencia (Medina, 2000).

La adultez se considera una etapa en la que se producen transformaciones psicológicas relevantes, un período de la vida (como en otras etapas) en el que se gana y se pierde, y donde, debido a la gran plasticidad de la edad, las pérdidas pueden quedar neutralizadas por las nuevas ganancias; una etapa, en suma, en la 
que, aunque no haya crecimiento, hay desarrollo y tiene sentido el aprendizaje que también puede ser optimizado mediante la educación (Medina, 2000).

El punto de partida es una concepción amplia del aprendizaje, comprendido como la capacidad del ser humano para anticiparse al futuro para resolver las situaciones nuevas, para arriesgarse a pensar, para seguir aprendiendo (Escotet, 1990, citado por Villanueva, 2001). En esta definición las concepciones lineales del aprendizaje se encuentran superadas, y se da énfasis a los múltiples aspectos que pueden incidir en el desarrollo de este complejo proceso.

En esta línea Villanueva (2001) estableció que los aspectos generales que inciden en el aprendizaje de las personas son tres:

- En primer lugar, aspectos provenientes de las teorías sobre psicología de la instrucción y psicología del aprendizaje que incorporan perspectivas tanto cognitivas como constructivistas, fundamentales para la comprensión de los diversos entramados que conforman la amplia red del aprendizaje del ser humano.

- Un segundo aspecto provendría de la influencia de determinados factores intrapersonales. Aquí entrarían en juego los estilos cognitivos de la persona, pero también otros factores de personalidad (ansiedad, expectativas de control y concepto de sí misma), la motivación del o de la aprendiente y, sobre todo, sus propias estrategias de aprendizaje y trabajo intelectual autónomo.

- Finalmente, aspectos resultantes de determinados factores socioambientales e interpersonales: los diversos escenarios educativos, el comportamiento de la persona mediadora y sus estilos de enseñanza, las expectativas del o de la docente y las interacciones docente-aprendiente y aprendiente-aprendiente. 
El cuadro 1 resume factores fundamentales que contemplan las perspectivas cognitiva y constructivista en el aprendizaje:

\begin{tabular}{|c|c|}
\hline Perspectiva cognitiva & Perspectiva constructivista \\
\hline $\begin{array}{l}\text { Estilos sensoriales: visual, auditivo y } \\
\text { kinestésico }\end{array}$ & $\begin{array}{l}\text { Proceso activo de elaboración de } \\
\text { significados }\end{array}$ \\
\hline $\begin{array}{l}\text { Estilos cognitivos: activo, teórico, } \\
\text { reflexivo y pragmático }\end{array}$ & $\begin{array}{l}\text { Asimilación y acomodación (Piaget) } \\
\text { Contexto y zona de desarrollo próximo } \\
\text { (Vygotsky) }\end{array}$ \\
\hline $\begin{array}{l}\text { Del pensamiento concreto al } \\
\text { pensamiento abstracto }\end{array}$ & $\begin{array}{l}\text { Cambios a partir de la re-significación } \\
\text { de nociones anteriores }\end{array}$ \\
\hline $\begin{array}{l}\text { Almacenamiento, codificación } \quad \text { y } \\
\text { recuperación }\end{array}$ & $\begin{array}{l}\text { Personal, subjetivo, afectivo, situado y } \\
\text { contextualizado }\end{array}$ \\
\hline $\begin{array}{l}\text { Atención y memoria (sensorial, trabajo y } \\
\text { largo plazo) }\end{array}$ & $\begin{array}{l}\text { Se construye con y a partir de otro; el } \\
\text { aprendizaje es social. }\end{array}$ \\
\hline $\begin{array}{l}\text { Influye tamaño, intensidad, novedad, } \\
\text { incongruencia, emoción y significado } \\
\text { personal }\end{array}$ & $\begin{array}{l}\text { Indagación, tutelaje, problemas, } \\
\text { colaboración, enseñanza recíproca }\end{array}$ \\
\hline
\end{tabular}

Fuente: Elaboración propia a partir de Alonso, Gallego y Honey (1994), Ellis (2005), Woolfolk (2010) y Zabalza (2007)

Medina (2000) también ha orientado sus investigaciones al reconocimiento de las características propias de la adultez que vendrían a incidir en el aprendizaje durante dicha etapa. Así distinguió cuatro aspectos particulares que deben considerarse:

a. Se debe contemplar el significado social de la adultez, ya que este otorga determinados roles socioculturales que rigen las demandas y la forma de comportarse de la persona en una determinada sociedad. 
b. Hay que partir de que dicho grupo etario resulta el más numeroso, si se le compara con la cantidad de personas que se incluyen en las etapas de la infancia y la adolescencia. Tanto es así que la adultez se subdivide en tres períodos diferenciados que se suceden a lo largo de la vida y difieren en el inicio y la finalización de esta etapa, tal como se señala en el cuadro 2 :

\begin{tabular}{|ccccccc|}
\multicolumn{6}{c|}{ Cuadro 2. Etapas y años de la adultez según diferentes autores } \\
\hline Etapas & $\begin{array}{c}\text { Erikson } \\
1981\end{array}$ & $\begin{array}{c}\text { Levinson } \\
1986\end{array}$ & $\begin{array}{c}\text { Papalia } \\
1992\end{array}$ & $\begin{array}{c}\text { Rice } \\
1997\end{array}$ & $\begin{array}{c}\text { Graig } \\
1997\end{array}$ & $\begin{array}{c}\text { Otras } \\
\text { denominaciones }\end{array}$ \\
\hline Juventud & $20-35$ & $17-40$ & $20-40$ & $\begin{array}{r}20- \\
40\end{array}$ & $\begin{array}{r}20- \\
40\end{array}$ & Adultez temprana \\
\hline $\begin{array}{c}\text { Mediana } \\
\text { edad }\end{array}$ & $35-65$ & $40-65$ & $40-65$ & $\begin{array}{r}40- \\
60\end{array}$ & $\begin{array}{c}40- \\
60\end{array}$ & $\begin{array}{c}\text { Adultez media, } \\
\text { madurez }\end{array}$ \\
\hline Vejez & $>65$ & $>65$ & $>65$ & $>60$ & $>60$ & $\begin{array}{c}\text { Adultez } \\
\text { avanzada, } \\
\text { ancianidad }\end{array}$ \\
\hline
\end{tabular}

Fuente: Elaboración propia a partir de Medina, 2000

c. En esta etapa también se desarrollan cambios biológicos importantes: la configuración orgánica que venía en un proceso de maduración entre los veinte y los treinta alcanza su punto más alto, por lo que la capacidad física empezará a disminuir en los años posteriores. A la vez, se presenta el fenómeno de la compensación, dado que "cualquier déficit, limitación o pérdida lleva en sí mismo la capacidad para generar nuevas formas de innovación y progreso» (Vega y Bueno, 1995, citados por Medina, 2000, p.99).

d. Los cambios cognitivos se van a ver afectados dependiendo de la concepción de inteligencia de la que se parta, por lo que es necesario trascender de una mirada de acumulación a una postura de autogobernabilidad personal y social. 
Hoy se reconoce que las capacidades intelectuales varían en la edad adulta, ya que se gana en algunos aspectos aunque se pierde en otros (inteligencia fluida versus cristalizada):

- En la vida adulta, además del pensamiento formal, se utilizan otras formas de pensamiento post formal (pensamiento práctico, pensamiento dialéctico, pensamiento divergente, pensamiento flexible, etc.). Así como el pensamiento depende del lenguaje, en esa misma medida el conocimiento y el aprendizaje dependerán de las relaciones y las interacciones sociales. En este sentido, el aprendizaje social (un aprendizaje dialógico basado en las habilidades comunicativas que poseen todos las personas) ocupa una posición sobresaliente en la edad adulta (Medina, 2000).

- Con respecto a la memoria, pareciera que algo se pierde con la edad, pero afectaría solo a un tipo de memoria y a algunos procesos. Así, la memoria sensorial y primaria no se ve afectada, pero sí la secundaria, especialmente en relación con el aprendizaje: se hace más selectiva, se pierde memoria de recuerdo, pero no para tareas de reconocimiento; se pierde memoria para los datos, fechas, hechos, nombres, detalles, anécdotas y otros, pero no para los significados, las ideas, las interpretaciones, el análisis y la síntesis (Palacios y Marchesi, 1991, citados por Medina, 2000).

Asimismo, a partir de los aportes de diferentes investigaciones (Bordignon, 2006; Medina, 2000; Picón, 2013; Ruiz, 2001 y Villanueva, 2001) se empiezan a esbozar otras características interesantes que aportan a la comprensión de los procesos de aprendizaje en esta etapa. A continuación se presentan algunos factores que sirven de base para la futura discusión y reflexión de la mediación pedagógica ante este panorama.

\section{Las tareas y el aprendizaje en la etapa adulta}

Según los aportes de Eric Erikson (1981, citado por Bordignon, 2006), la tarea fundamental en la etapa adulta, a nivel del desarrollo psicosocial, se encuentra 
relacionada con lograr la intimidad en las diferentes esferas de su medio. Visto así, la intimidad sería

La fuerza sintónica que lleva al joven adulto a confiar en alguien como compañero en el amor y en el trabajo, integrarse en afiliaciones sociales concretas y desarrollar la fuerza ética necesaria para ser fiel a esos lazos, al mismo tiempo que imponer sacrificios y compromisos significativos (p.57).

Así, un justo equilibrio entre la intimidad y el aislamiento fortalecería la capacidad de la realización, la cual se expresaría en las relaciones sociales de integración y los compromisos en instituciones y asociaciones culturales, políticas, deportivas y religiosas. Dado este proceso psicosocial, se considera que «más allá de una programación estructurada institucionalmente, los sucesos evolutivos, tales como la entrada en el mundo laboral, la relación de intimidad, el matrimonio, la paternidad o la jubilación, se convierten en situaciones de aprendizaje propias y específicas del adulto de nuestra sociedad» (Ruíz, 2001, párr. 30).

De esta manera, dichos sucesos actúan como situaciones de aprendizaje significativo en las cuales la persona requiere desarrollar nuevas estrategias que transfiere a otras áreas de su vida, poniendo en marcha estrategias de tipo cognitivo, pero también desarrollando actitudes, valores y conductas propias por el paso y la vivencia de dichos sucesos. Esta sería la principal razón para que los sucesos evolutivos sean aprovechados en el desarrollo cognitivo adulto como reactivos que muestran la capacidad adaptativa, la solución de problemas o la utilización de estrategias nuevas ante contextos conocidos, superando lo aprendido previamente.

El aprendizaje significativo se comprendería, entonces, como aquel en el que la persona, a partir de sus conocimientos previos (preconcepto) y favorecida por la mediación (interacción), reorganiza sus conocimientos (esquemas cognitivos) con 
nuevas dimensiones y estructuras (subordinación, supraordinación, etc.), lo cual le permite ser capaz de realimentarse de otras realidades (funcionalidad cognitiva), de describir los procesos y principios explicativos que afectan tales realidades (significatividad lógica) y de mejorar su capacidad de organización comprensiva (aprender a aprender) en relación con otras experiencias de aprendizaje (significatividad psicológica) (Ruíz, 2001). Lo anterior indicaría que los sucesos evolutivos se convierten en las tareas de aprendizaje significativo más importantes en el desarrollo adulto, lo cual obligaría a las personas que median estos procesos a repensar las estrategias a utilizar en función de mejorar las ecologías cognitivas de sus aprendientes.

\section{La cognición y el aprendizaje en la etapa adulta}

No se puede negar que, en cuanto al aprendizaje, la infancia, la adolescencia y la adultez tienen algunos procesos cognitivos similares; sin embargo, como ya se ha indicado, para el fin de este texto interesa destacar las diferencias cualitativas que se producen durante la edad adulta. No obstante, debe reconocerse que las investigaciones sobre el aprendizaje en esta etapa son pocas, pues la mayoría de estudios giran en torno a los procesos cognitivos que se desarrollan en la infancia, y en el caso de que sí se considere la adultez, muchas veces brindan pocas luces para la comprensión de los procesos que sustentan el aprendizaje en esta edad (Medina, 2000).

Dentro de las investigaciones realizadas lo que sí puede señalarse es que «el tipo de aprendizaje que se produce en la edad adulta, donde la experiencia y la inteligencia cristalizada juegan un papel fundamental, no es equiparable al que se produce en la edad infantil (época en la que las operaciones concretas ocupan un lugar relevante)»(Medina, 2000, p. 101). Ahora bien, cuando se ha comparado la capacidad de personas mayores con la de las más jóvenes, se manifiesta que las diferencias dependen no tanto de la edad, sino más bien de factores relacionados 
con la naturaleza de las pruebas o con las propias características de cada persona.

A pesar de lo anterior, algunos autores han comprendido las necesidades específicas que tienen las personas que educan población adulta, y han asumido el reto de aportar elementos característicos de lo que podría ser una pedagogía sobre el aprendizaje de este grupo.

En primer lugar, vale la pena referirse a la construcción que la persona adulta madura realiza de su realidad: las habilidades conllevan cambios en la relación que mantiene con la realidad como objeto de conocimiento. En palabras de Labouvie-Vief (1992, p. 209): «El sujeto maduro llega a comprender el conocimiento como una transformación social, activa, de la realidad a través de un proceso de cuestionamiento crítico que tiene en cuenta el contexto histórico» (citado por Ruiz, 2001, párr. 7).

Esto repercutiría en que los dualismos cognoscitivos presentes en la población adolescente (leyes objetivas científicas frente al mundo subjetivo del propósito y de la creencia), tras una posición de relativismo radical («nada se puede conocer objetivamente; la realidad solo es objeto de conocimiento personal»), pueden llegar a integrarse en una forma más abstracta de subjetivismo: «En este punto el sujeto se da cuenta de los límites de un puro concepto externo de lo que es "objetivo" y trata de volver a recolocar los criterios de verdad, no ya en la esfera personal, sino más bien en la interpersonal» (Ruiz, 2001, párr. 8).

Este balance entre lo objetivo y lo subjetivo apoya una estructura superior que se traduce en un instrumento eficaz para la autocorrección de los propios procesos cognitivos, tal y como lo caracterizó Ruiz (2001): 
a) En el análisis de problemas sociomorales aumenta la capacidad para elaborar versiones más equilibradas y menos sesgadas de informaciones discrepantes sobre el mismo hecho.

b) Aparece una nueva regulación cognitiva denominada cognición epistémica, preocupada por las condiciones del conocimiento: sus límites, el nivel de certeza que es posible alcanzar, etc.

c) Se está menos preocupado por la resolución de problemas que por el hecho de descubrirlos. Se presenta un estadio del descubrimiento divergente, la formulación de problemas genéricos, el aislamiento y la resolución de problemas mal definidos y el proceso de pensamiento progresivo.

En congruencia con las habilidades que se desarrollan en la etapa de la adultez, resulta fundamental que las experiencias de aprendizaje también se encaminen a reencontrar a la persona con el conocimiento de manera significativa, próxima y reflexiva. En ese sentido, diversas investigaciones proponen una selección de principios encaminados a mejorar el aprendizaje en la edad adulta (Medina, 2000 y Zabalza, 2007):

a) El aprendizaje está condicionado por todo el conjunto de capacidades y habilidades al que recurren las personas aprendientes. Los adultos necesitan tener la oportunidad de expresar lo que saben.

b) El aprendizaje es producto de la práctica, del tiempo de trabajo que se solicite realizar y de las condiciones en las que se encuentra. El ritmo de ejecución de las tareas debe permitir un desempeño óptimo, dado que se valora más la precisión en el desempeño que la rapidez.

c) La percepción de la tarea, de los procesos instructivos y de las atribuciones causales de los resultados (externas-internas) afectan el propio aprendizaje. La motivación mejora cuando se comprende la relevancia o la aplicación de lo que se ha de aprender. 
d) El aprendizaje se ve también condicionado por la particular «negociación de expectativas» que se produce entre los protagonistas del proceso. El desempeño de las personas adultas es mayor con tareas que resulten relevantes y posean sentido para ellas.

e) Tienen notable importancia en el aprendizaje la atención, la implicación personal y la realimentación. Es necesario eliminar la ansiedad en relación con la eficiencia y los resultados.

f) La información que se organiza en categorías, con secuencias lógicas y agrupadas en asociaciones significativas, a través de varios sentidos y sin estar fatigado, promueve o facilita el aprendizaje.

Ahora, si bien estos principios metodológicos pueden brindar algunas pistas para el fortalecimiento de contextos de aprendizaje más flexibles, no son suficientes para alcanzar el carácter optimizante que debe prevalecer en la acción educativa con personas adultas. De este modo, resulta importante retomar otras propuestas más elaboradas acerca del aprendizaje en la edad adulta que ponen sobre la mesa aspectos medulares del sentido mismo de la educación: su intencionalidad y reflexión crítica.

En esta línea cabe rescatar la propuesta de Freire (1992) sobre la educación liberadora como un tipo de educación que se opone a la educación bancaria (proceso en el que la persona educadora deposita contenidos en la mente del educando). En la primera el aprendizaje resulta emancipador (liberador, optimizador) tan solo si la acción educativa es problematizadora y esperanzadora, lo cual se logra por medio de un diálogo entramado entre los y las aprendientes (educadores y educandos):

Conviene destacar que el interés de la propuesta de Freire sobre el aprendizaje dialógico no solo reside en las ventajas que tiene el diálogo como un medio efectivo para el aprendizaje (...) más importante resulta todavía el modo en que sitúa el diálogo (y sus 
características) en el centro de gravedad de los procesos de aprendizaje, como condición ineludible para que la acción educativa sea crítica y transformadora (Medina, 2000, p.107).

Este cambio de mirada ( $\mathrm{y}$ de vínculos) trasciende de relaciones de poder verticales a espacios descentralizados horizontales, y, en este sentido, se requiere humildad por parte de la persona educadora para descubrir la sabiduría del otro:

No hay (...) diálogo si no hay humildad. La pronunciación del mundo, con el cual los hombres lo recrean permanentemente, no puede ser un acto arrogante. Freire se opone a toda arrogancia y a la separación tajante entre los participantes en un proceso educativo: «La educación debe comenzar por la superación de la contradicción educador-educando. Debe fundarse en la conciliación de sus polos, de tal manera que ambos se hagan, simultáneamente, educadores y educandos (Freire, 1992, p. 77)» (Santos, 2008, p.159).

Este aprendizaje dialógico es global y se basa en la comunicación y en el lenguaje como medio de entendimiento; además es un tipo de aprendizaje válido para una gran diversidad de contextos. El aprendizaje de las personas adultas tendrá efectos emancipadores si, por una parte, se concibe que ellas mismas son capaces de conocer, aprender y participar y, por otra parte, se utiliza el diálogo entre las personas participantes como el medio más adecuado para el desarrollo del conocimiento, la adquisición de competencias y el cambio social (Ayuste, 1999, citada por Medina, 2000).

\section{La personalidad y el aprendizaje en la etapa adulta}

Como se ha evidenciado a lo largo de este artículo, en la edad adulta existe una serie de condiciones suficientes que permitirían el autoaprendizaje, entre las cuales se destaca la autonomía, la toma de decisiones y la capacidad para asumir compromisos, las características más favorecedoras en dicho proceso. 
Los principales rasgos de personalidad que caracterizan la etapa de la adultez pueden apoyar la relación y consecución de experiencias de aprendizaje. Sobre esto Medina (2000) ha establecido algunos elementos que, en la medida en la que se presenten en la persona, pueden potenciar su desarrollo:

a) Estabilidad emocional: Se supondría una madurez y control para afrontar las adversidades y ajustarse a las inevitables frustraciones del entorno.

b) Autonomía para la toma de decisiones y para la acción: al romper los vínculos de dependencia y avanzar hacia una mayor independencia en sus juicios y libertad en la toma de decisiones

c) Identidad personal: Supone un incremento del conocimiento de sí mismo, lo cual permite avanzar a la definición de rasgos propios que facilita la intimidad con otros.

d) Responsabilidad: Se logra el desarrollo racional y de autonomía encomendado socialmente.

e) Participación social: Se aprenden normas y valores morales y sociales, se comparten experiencias y sentimientos y se responde a los requerimientos de grupo de referencia. Esta forma de vincularse promueve la satisfacción, el compromiso y la solidaridad.

Según estos rasgos, las experiencias de aprendizaje no solo partirían del supuesto de que las personas aprendientes cuentan con estas características, apoyándose en estas premisas para mediar y evaluar el proceso, sino que también son potenciadoras de los mismos rasgos para que la persona logre desarrollar una plena identidad por medio de su ser, hacer y conocer.

\section{La experiencia de aprendizaje en la etapa adulta: ¿Cómo mejorarla?}

Todos estos hallazgos invitan a repensar el papel de la mediación pedagógica y los retos que enfrenta en la educación a distancia cuando las personas participantes se encuentran inmersas en complejos ámbitos (trabajo, familia, 
amigos, ocio) y, a la vez, presentan características biopsicosociales propias, definidas por la etapa de desarrollo en la que se encuentran.

El punto de partida para re-pensar las experiencias de aprendizaje en la adultez resulta del reconocimiento del carácter propio que posee esta etapa. Así, las personas adultas se conciben como constructoras permanentes de su aprendizaje, lo que significa que tienen la capacidad de conocer y de actuar mejorando sus propias condiciones personales y sociales. Esto no se puede lograr sin que se dé una relación más horizontal entre los y las protagonistas que intervienen en este proceso.

Otra condición inherente en el proceso de aprendizaje es que las personas confíen en sus propias capacidades. En este sentido, no resulta tan novedoso que dicha imagen sea reforzada por el concepto que tiene el mediador del estudiante y, más allá, por la forma en la que le realimenta, se comunica y genera espacios de diálogo e interacción (formal y no formal). Visto de esta manera, la planificación de las enseñanzas programáticas no será más importante que las propias atribuciones de los y las aprendientes, pues finalmente estas incidirán directamente en su proceso de formación (Woolfolk, 2010).

Una vez establecida esta base de confianza la actividad vendría a constituir una oportunidad para la generación de experiencias, siempre y cuando estas faciliten la construcción de aprendizajes significativos (gestores de rupturas y cambios). Por ello, se recomienda que dichas experiencias incorporen construcciones con sentido: mapas mentales o conceptuales, resolución de un problema, proyectos, planes, investigaciones, producciones audiovisuales o artísticas, afiches, etc., así como su integración y realimentación en los espacios grupales.

Para el desarrollo adecuado de las actividades de aprendizaje hace falta un ingrediente básico: la motivación, un estado interno que activa, dirige y mantiene 
la conducta y, por consiguiente, la atención. De esta manera, una persona con una alta motivación para aprender se toma en serio el trabajo académico, trata de obtener lo mejor de este y aplica estrategias de aprendizaje adecuadas durante el proceso:

Las tareas que asigna el profesor influyen en la motivación. Cuando los estudiantes se enfrentan a tareas que se relacionan con sus intereses, que estimulan su curiosidad o que están relacionadas con situaciones de la vida real, tienden a estar más motivados para aprender. Las tareas tienen un valor de logro (importancia) intrínseco (gozo) o utilitario (metas) para los estudiantes. El valor de logro es la importancia que tiene el éxito para el alumno (Woolfolk, 2010, p. 210).

Ahora bien, aunque la motivación sea elevada, el aprendizaje no se consolida ni se hace duradero si la información (los contenidos del aprendizaje) no llega a procesarse convenientemente. Por esto es importante organizar la experiencia para que pueda ser procesada, almacenada o construida de la mejor manera, tal como se ejemplifica en el cuadro 3 :

\begin{tabular}{|ll|}
\hline \multicolumn{2}{|c|}{$\begin{array}{c}\text { Cuadro 3. Aprendizaje profundo y las prácticas tradicionales } \\
\text { Aprendizaje profundo del conocimiento } \\
\text { (hallazgos de las ciencias } \\
\text { cognoscitivas) }\end{array}$} \\
\hline $\begin{array}{l}\text { Requiere que los y las aprendices } \\
\text { relacionen los conceptos y las ideas } \\
\text { nuevas con los conocimientos y } \\
\text { experiencias anteriores. }\end{array}$ & $\begin{array}{l}\text { Los y las aprendices consideran que } \\
\text { el material del curso no está }\end{array}$ \\
\hline $\begin{array}{l}\text { Requiere que los y las aprendices integren } \\
\text { sus conocimientos en sistemas } \\
\text { conceptuales interrelacionados. }\end{array}$ & Los y las aprendices consideran el \\
\hline Demanda que los y las aprendices & material del curso como fragmentos \\
\hline
\end{tabular}


busquen patrones y principios

subyacentes.

Solicita que los y las aprendices evalúen

ideas nuevas y que las relacionen con

conclusiones. hechos y realizan procedimientos sin entender el cómo ni el porqué.

A los y las aprendices les resulta difícil darles sentido a ideas nuevas que difieren de lo que encuentran en las lecturas del curso.

Requiere que los y las aprendices comprendan los procesos de diálogo que crean el conocimiento y que puedan examinar la lógica de un argumento de manera crítica.

Demanda que los y las aprendices Los y las aprendices memorizan sin reflexionen acerca de su propia reflexionar acerca del objetivo o de comprensión y de su propio proceso de aprendizaje. sus propias estrategias de aprendizaje.

Utiliza organizadores estructurales (de qué se trata), semántico-conceptuales (conceptos claves), de sentido (para qué sirven) y personales (implicación propia y conocimientos previos).
Los y las aprendices consideran los hechos y los procedimientos como conocimientos estáticos, transmitidos por una autoridad omnisciente.
Los y las aprendices indican que no encuentran una organización de la experiencia, ya que no comprenden el sentido y alcance de lo que se les presenta.

Fuente: Elaboración propia a partir de Woolfolk (2010) y Zabalza (2007)

Este posicionamiento lleva a entender el aprendizaje en la edad adulta, más que como un proceso de aculturación, como un encuentro de culturas entre aprendientes (incluyendo a la persona mediadora del espacio de aprendizaje), pues las personas adultas se enfrentan a dichos procesos desde una experiencia que han acumulado durante años. En virtud de estos antecedentes, las personas adultas tienen unos comportamientos consolidados, poseen una personalidad madura y estructurada, tienen responsabilidades familiares y laborales y aprecian 
lo que consideran valioso y verdadero. Inclusive, cuentan con unos conocimientos y habilidades que, a veces, no poseen ni los propios profesores (Medina, 2000).

En este sentido, el encuentro de aprendientes se ve direccionado por la experiencia como fuente de aprendizaje y de conocimiento. Es por ello que la experiencia tiene un rol fundamental: facilita el procesamiento de la información al permitir establecer relaciones significativas entre los nuevos aprendizajes y los que ya se poseen; funciona como fuente de aprendizaje y conocimiento, siempre y cuando considere la experiencia previa; ayuda a comparar los nuevos conocimientos con la práctica y con la experiencia propia, lo que los hace ser más críticos. En otras palabras, ayuda a construir el conocimiento (ver cuadro 4):

\begin{tabular}{|c|c|c|}
\hline Tipo & $\begin{array}{l}\text { Supuestos acerca del aprendizaje y los } \\
\text { conocimientos }\end{array}$ & $\begin{array}{l}\text { Ejemplos de } \\
\text { teorías }\end{array}$ \\
\hline $\begin{array}{l}\text { Dirección } \\
\text { externa }\end{array}$ & $\begin{array}{l}\text { Los conocimientos se adquieren al construir una } \\
\text { representación del mundo externo. La enseñanza } \\
\text { directa, la retroalimentación y la explicación afectan } \\
\text { el aprendizaje. Los conocimientos son precisos de } \\
\text { acuerdo con el grado en el que reflejan la «forma } \\
\text { en que realmente son las cosas» en el mundo } \\
\text { externo. }\end{array}$ & $\begin{array}{l}\text { Procesamiento de } \\
\text { la } \\
\text { información }\end{array}$ \\
\hline $\begin{array}{l}\text { Dirección } \\
\text { interna }\end{array}$ & $\begin{array}{l}\text { Los conocimientos se construyen al transformar, } \\
\text { organizar y reorganizar conocimientos previos. Los } \\
\text { conocimientos no son un espejo del mundo } \\
\text { exterior, aun cuando la experiencia afecta el } \\
\text { pensamiento, y este afecta los conocimientos. La } \\
\text { exploración y el descubrimiento son más } \\
\text { importantes que la enseñanza. }\end{array}$ & Piaget \\
\hline
\end{tabular}




\section{Dirección Los conocimientos se construyen con base en las Vygotsky interna interacciones sociales y en la experiencia. Los \\ y externa conocimientos reflejan el mundo externo, filtrado e influido por la cultura, el lenguaje, las creencias, las interacciones con los demás, la enseñanza directa $y$ el modelamiento. El descubrimiento guiado, la enseñanza, los modelos y el entrenamiento, así como los conocimientos previos, las creencias y el pensamiento del individuo, afectan el aprendizaje.}

Ahora bien, en la construcción del conocimiento entran en juego diversas estrategias, y en la edad adulta el aprendizaje se realiza sobre problemas:

No tiene sentido en la edad adulta un aprendizaje meramente académico y formal basado en las materias o asignaturas clásicas. Más bien el aprendizaje hay que concebirlo como un medio, para abordar y enfrentarse mejor a los problemas de su vida cotidiana, familiar, social y laboral (Medina, 2000, p.111).

Las características biopsicosociales de la persona adulta favorecen el aprendizaje dialógico, en el que la participación y el diálogo son medios privilegiados y posibilitadores de la adquisición de compromisos sociales, del mejoramiento de la autoestima y del incremento de la motivación que, sumados al autoaprendizaje, facilitarían la autonomía para la toma de decisiones y la capacidad para asumirlas responsablemente: «En todo caso, el equilibrio en la combinación de heteroenseñanza, por un lado, y autoaprendizaje, por el otro, es lo que podría aportar altos rendimientos para el aprendizaje de adultos» (Medina, 2000, p. 112).

Asimismo, la realimentación es un elemento fundamental del proceso de aprendizaje; puede ser llevada a cabo por la propia persona, el profesor o la 
profesora, los compañeros y las compañeras o las familias. A nivel cognitivo sirve como indicación y guía del camino a seguir, puesto que ofrece información sobre la actividad desarrollada y su pertinencia. En lo afectivo lleva a vivenciar sentimientos de éxito o fracaso, pero también sirve de expresión de la presencia y apoyo de los otros, más en el caso de las personas que median el proceso (Zabalza, 2007).

Finalmente, debe considerarse que, por las características asociadas al ciclo vital de esta etapa, las personas aprendientes se encuentran inmersas en otros roles: son personas casadas, padres, empleados, empresarios, líderes, etc., y, secundariamente, son estudiantes. Consecuentemente, las experiencias de aprendizaje deben, primero que todo, relacionarse con estos contextos $y$, después, no ocupar más tiempo del que merecen. Así las cosas, se trata de brindar espacios de calidad y no de cantidad.

\section{Conclusiones}

Las características de la población que ingresa a la UNED han cambiado; así también las condiciones del entorno y las necesidades laborales y sociales que acompañan a una sociedad posindustrial que se configura desde la globalidad, el cambio y la incertidumbre. En esta misma línea, los esfuerzos de la academia deben orientarse hacia la construcción de espacios que guíen significativamente a estos ciudadanos para su desenvolvimiento integral con sus familias y en sus trabajos y comunidades.

Las personas jóvenes y adultas tienen algunas situaciones comunes, y, aunque pedagógicamente pueden compartir algunos aprendizajes, es un hecho que el aprendizaje contextualizado de cada categoría tiene unas especificidades que deben ser adecuadamente atendidas: "De otro lado, las niñas y niños y las y los adolescentes tienen sus propios universos de intereses estratégicos y de 
motivaciones $y$, por tanto, sus formas de aprendizaje tienen que ser necesariamente diferenciadas" (Picón, 2013, p.390).

Así pues, se puede afirmar con propiedad que cuando se habla de la educación de personas adultas se está ante una teoría educativa específica, cuyas características son propias de un tipo de educación social. Por esta razón, en la universidad debe destacar un modelo de educación que atienda no solo la profesionalización, sino también la formación de ciudadanos y ciudadanas con valores éticos y actitudes generadoras de una convivencia social que favorezca el desarrollo humano de niños, niñas, adolescentes y adultos y adultas mayores.

Una concepción socioconstructivista del aprendizaje demanda una intervención diferente de quienes median el aprendizaje. Si bien el aprendiente es el responsable último de su proceso, la persona docente no puede limitarse únicamente a crear las condiciones óptimas para el despliegue de una actividad mental constructiva y diversa. Más aún, su tarea es guiar y orientar esta actividad, con el fin de que la construcción del y de la aprendiente se acerque progresivamente a lo que significan y representan los contenidos, y la relación de estos con sus diferentes nichos vitales, de forma que se configuren las llamadas ecologías cognitivas (Assman, 2002). En este sentido, la relación aprendienteprofesor en los espacios profesionalizantes resulta fundamental, porque lo que hace la persona adulta es reconstruir en su mente los contenidos culturales acumulados a lo largo de su experiencia de vida, y es el profesor el que actúa de intermediario cualificado entre los contenidos de aprendizaje y la actividad constructiva que despliega la persona aprendiente para asimilarlos (Villanueva, 2001).

El aspecto instructivo de la educación superior actual no consigue dar cuenta de la profusión de conocimientos disponibles y emergentes, incluso en áreas específicas. Así, no debería preocupar tanto la memorización de los saberes 
instrumentales, sino el desarrollo de la capacidad de "acceder a ellos", decodificarlos y manejarlos: "El aspecto de instrucción debería estar en función del aprendizaje emergente (emergent learning), es decir, de la morfogénesis personalizada del conocimiento" (Assmann, 2002, p.32).

Los aportes teóricos mencionados marcan la necesidad de establecer nuevas formas de mediar los aprendizajes con las personas adultas; así

Resulta preciso sustituir la pedagogía de las certezas y de los saberes prefijados por una "pedagogía de la pregunta", de la mejora de las preguntas y del "acceso" a las informaciones. En suma, por una pedagogía de la complejidad, que trabaje con conceptos transversales, abiertos a la sorpresa y a lo imprevisto (Assmann, 2002, p.32).

Este aprendizaje debe ser integral, y no una mera adquisición de conocimientos para el desempeño de una profesión. Además, requiere el trabajo con ámbitos cognoscitivos, pero no exclusivamente, ya que el desarrollo de competencias que implican destrezas, habilidades y actitudes para la convivencia y el desarrollo social se convierte en tarea prioritaria. Cualquier tipo de educación, en cualquiera de sus niveles, debe prestar atención por igual a dos facetas que deben ir inseparablemente unidas: la faceta instructiva o dimensión de la enseñanza, entendida como transmisión de cultura, conocimientos, disciplinas y programas; y la faceta formativa o de desarrollo y transformación de actitudes, valores, hábitos, y comportamientos que deben interrelacionarse a lo largo del proceso de aprendizaje (García, 2008).

Dado el carácter re-constructor y transformador de la mediación pedagógica con personas adultas, el docente universitario debe perfilar sus funciones dentro del rol de tutor; esto le posibilitará acompañar a los y las aprendientes en diversos procesos: encontrar sentido de unidad a su aprendizaje, al integrar diferentes componentes (conocimientos, actitudes, competencias, hábitos, destrezas...); 
brindar globalidad e integralidad a la formación académica, científica y técnica; generar experiencias de aprendizaje que encaminen hacia la madurez personal y el crecimiento intelectual y científico; favorecer la formación de un espíritu, estilo y perfil universitario; apoyar los estilos y modos de aprendizaje; $y$, finalmente, servir de referente y apoyo, de maestro guía (García, 2008).

De lo anterior se deduce la necesidad de abordar los procesos de planificación de la enseñanza desde otra perspectiva, según la cual las personas mediadoras no centran su tarea en la transmisión de conocimientos, sino en ser gestoras de procesos de aprendizaje contextualizados a las características, las necesidades y los intereses de la edad adulta. Lo anterior implica centrarse fundamentalmente en ofrecer herramientas y medios para que sea la persona aprendiente quien, de forma autónoma, controle su propio proceso de aprendizaje y aplique estos saberes en sus nichos vitales, brindando además el acompañamiento necesario para superar la dificultades que encuentre en sus rutas de aprendizaje (De Miguel, 2005).

Para concluir, resulta útil destacar las palabras de Marín Ibáñez (1977, p. 115):

El papel del profesor cambia también en este tipo de enseñanza. Ya no es quien posee todo el saber, que imparte a un pasivo alumno (...) ahora es el calificado colaborador del aprendizaje, ayuda al alumno a diseñar la marcha del trabajo, le facilita estímulos, ofrece materiales, prepara las experiencias más adecuadas y contribuye a clarificar los objetivos (citado por Medina, 2000, p.136).

En el legado intelectual de este maestro la configuración de la función de la mediación va más allá de un mero cambio técnico y metodológico para proyectarse en un verdadero compromiso con el cambio social. 


\section{Referencias}

Alonso, C. M., Gallego, D. J. y Honey, P. (1994). Los Estilos de Aprendizaje. Bilbao, España: Ediciones Mensajero.

Assmann, H. (2002). Placer y Ternura en la Educación. Madrid, España: Narcea.

Bordignon, N. (2006). El desarrollo psicosocial de Eric Erikson. El diagrama epigenético del adulto. Revista Lasallista de Investigación, 2 (2), 50-63.

Capra, F. (1992). El punto crucial. Buenos Aires, Argentina: Troquel.

De Miguel, M. (2005). Cambio de paradigma metodológico en la Educación Superior. Exigencias que conlleva. Cuadernos de Integración Europea, 2005 (2), 16-27. Recuperado de http://cde.uv.es/documents/2005-02-16.pdf

Ellis, J. (2005). Aprendizaje humano. Madrid, España: Pearson Educativa.

Freire, P. (1992). Pedagogía del Oprimido. Madrid, España: Siglo XXI.

García, N. (2008). La función tutorial de la Universidad en el actual contexto de la Educación Superior. Revista Interuniversitaria de Formación del Profesorado, 22 (1), 21 - 48.

McLaren, P. (1997). Pedagogía crítica y cultura depredadora. Políticas de oposición en la era posmoderna. Barcelona, España: Paidós Educador.

Medina, O. (2000). Especificidad de la educación de adultos. Bases psicopedagógicas y señas de identidad. Educación XXI, 2000 (3), 91-140.

Morin, E. (2006). El método 5: La humanidad de la humanidad. Madrid, España: Cátedra

Najmanovich, D. (2005). El Juego de los vínculos. Buenos Aires, Argentina: Biblos.

Picón, C. (2013). Educación de adultos en América Latina y el Caribe: utopías posibles, pasiones y compromisos. Michoacán, México: Centro de Cooperación Regional para la Educación de Adultos en América Latina y el Caribe.

Ruíz, C. (2001). ¿Cómo aprenden los adultos? Características del pensamiento. Revista de Educación a Distancia, 2001 (1). Recuperado de http://revistas.um.es/red/article/view/25151/24421 
Santos, M. (2008). Ideas filosóficas que fundamentan la pedagogía de Paulo Freire. Revista Iberoamericana de educación, 2008 (46), 155-173.

Sistema de Administración de Estudiantes (SAE). (2014). Estadísticas de matrícula 2010-2014 UNED [Informe digital]. Centro de Investigación y Evaluación Institucional (CIEI). San José, Costa Rica.

Villanueva, J. D. (2001). El aprendizaje de los adultos. Medicina de Familia, 2001 (2), 165-171.

Woolfolk, A. (2010). Psicología Educativa. México: Prentice Hall.

Zabalza, M. (2007). La enseñanza universitaria. El escenario y sus protagonistas. Madrid, España: Narcea. 